\title{
Incidences des procédés d'épuration appliqués aux effluents industriels sur le comportement chimique du ruthénium 106 dans l'environnement marin. Cas des rejets de l'usine de retraitement des combustibles irradiés de La Hague
}

\author{
R. GANDON*, Y. BARON**, A. FRAIZIER*, D. BOUST*, \\ P. GERMAIN* et P. GUÉGUÉNIAT**
}

(Manuscrit reçu le 25 janvier. 1994)

RÉSUMÉ Depuis la mise en service de l'usine de retraitement des combustibles irradiés de la Hague en 1966, l'efficacité des moyens employés pour épurer les effluents radioactifs liquides a été multipliée par un facteur 600 . Le comportement chimique du ${ }^{106}$ Ru déterminé dans les rejets déversés en mer et en deux stations d'observation proches de l'émissaire, a évolué parallèlement à l'évolution des procédés d'épuration. La méthodologie de type "voie humide" basée sur l'entraînement de ${ }^{106}$ Ru par le sulfure de cobalt a permis de répondre aux objectifs de protection de l'environnement et aux impératifs de production. Le comportement du radionucléide a alors été celui des complexes du nitrosylru. thénium chimiquement peu réactifs, générés au cours de la mise en solution des combustibles en milieu nitrique. A partir de 1989, la vitrification des effluents les plus marqués par ce type de complexation et l'optimisation du procédé par voie humide ont permis de limiter les rejets liquides de ${ }^{106} \mathbf{R u}$. Parallèlement, l'inertie chimique du radionucléide s'est atténuée. Les mesures de radioactivité de l'algue Fucus serratus déterminées in situ montrent que la disponibilité de ${ }^{106} \mathrm{Ru}$ à partir de l'eau de mer dépend étroitement de l'hydrolyse des complexes du nitrosylruthénium. Les produits d'hydrolyse du radionucléide paraissent être les formes privilégiées des échanges avec l'algue pour laquelle le facteur de concentration est évalué à $500 \pm 350$.

ABSTRACT Since nuclear fuel reprocessing started at the La Hague plant in 1966, the efficiency of the purification techniques applied to radioactive wastes has been improved by a factor of 600 . The chemical behaviour of ${ }^{106} \mathrm{Ru}$ observed in the liquid releases into the sea and at two coastal sites near the outlet, shows time

\footnotetext{
* Institut de protection et de sûreté nucléaire (IPSN), Département de protection de l'environnement et des installations (DPEI/ SERE/LRM), BP 10, 50130 Octeville.

** Groupe d'études atomiques de la marine nationale (GEA), BP 19, 50115 CherbourgNaval.
} 
variations related to the evolution of the reprocessing techniques used. The "wet chemical method" based on 106Ru coprecipitation with cobalt sulphide has fulfilled the objectives of environmental protection and the requirements of industrial productivity. As a consequence, ${ }^{106} \mathrm{Ru}$ behaves like the weakly reactive complexes formed during the nitric acid dissolution stage of the process. Since 1989 , vitrification of the effluents mainly affected by these complexes and the optimisation of the wet method have contributed to the reduction of ${ }^{106} R u$ liquid releases into the sea. At the same time, the radionuclide chemical nonreactivity has been attenuated.

Measurements of ${ }^{106}$ Ru activity carried out in the seaweed Fucus serratus show that the availability of nitrosylruthenium in seawater is closely related to hydrolysis of the complexes. Hydrolysis products are the main source of the chemical species available for exchange with the seaweed, its concentration factor being estimated at $500 \pm 350$.

\section{Introduction}

Le ruthénium est un des principaux éléments produits lors de la fission des noyaux lourds. La grande diversité de ses états de valence et de ses propriétés chimiques expliquent l'intérêt qu'il suscite en matière de radioprotection $[3,14]$. Il comporte seize isotopes, stables ou radioactifs, de nombre de masse compris entre 93 et $108 ;{ }^{106} \mathrm{Ru}$ est l'isotope radioactif de plus longue période (369 j). C'est un émetteur bêta pur. Son descendant, le rhodium 106 (30 s de période) est également un émetteur bêta accompagné de l'émission d'un rayonnement gamma. L'équilibre séculaire du couple ${ }^{106} \mathrm{Ru}-\mathrm{Rh}$ est obtenu en quelques minutes.

L'isotope ${ }^{106} \mathrm{Ru}$ est obtenu par fission thermique de ${ }^{235 \mathrm{U} \text { et }}{ }^{239} \mathrm{Pu}$. Les rendements de fission sont plus faibles que ceux obtenus pour les isotopes non radioactifs; ainsi, la contribution en masse de ${ }^{106} \mathrm{Ru}$ par rapport à l'ensemble des isotopes du ruthénium formés au cours des réactions nucléaires ne dépasse pas $1 \%$.

Depuis la signature, en 1963, du traité interdisant les essais nucléaires atmosphériques, le ${ }^{106} \mathrm{Ru}$ détecté dans l'environnement provient essentiellement des usines de retraitement des combustibles irradiés utilisant le procédé PUREX. Le ${ }^{106} \mathrm{Ru}-\mathrm{Rh}$ décelable dans les eaux marines du Nord-Cotentin provient principalement des rejets d'eaux résiduaires effectués par l'usine française de La Hague (Fig. 1). Il est à signaler que les retombées atmosphériques de l'accident de Tchernobyl survenu en 1986 n'ont pas significativement affecté ce secteur maritime de la Manche [26].

Depuis la mise en service de l'usine de La Hague en 1966 et jusqu'en 1990, ${ }^{106} \mathrm{Ru}-\mathrm{Rh}$ a contribué pour près de $75 \%$ à l'ensemble des émetteurs bêtagamma (hors tritium) rejetés en mer. La volonté de limiter ces rejets s'est donc traduite par une recherche de procédés d'épuration efficaces pour ${ }^{106} \mathrm{Ru}$. 

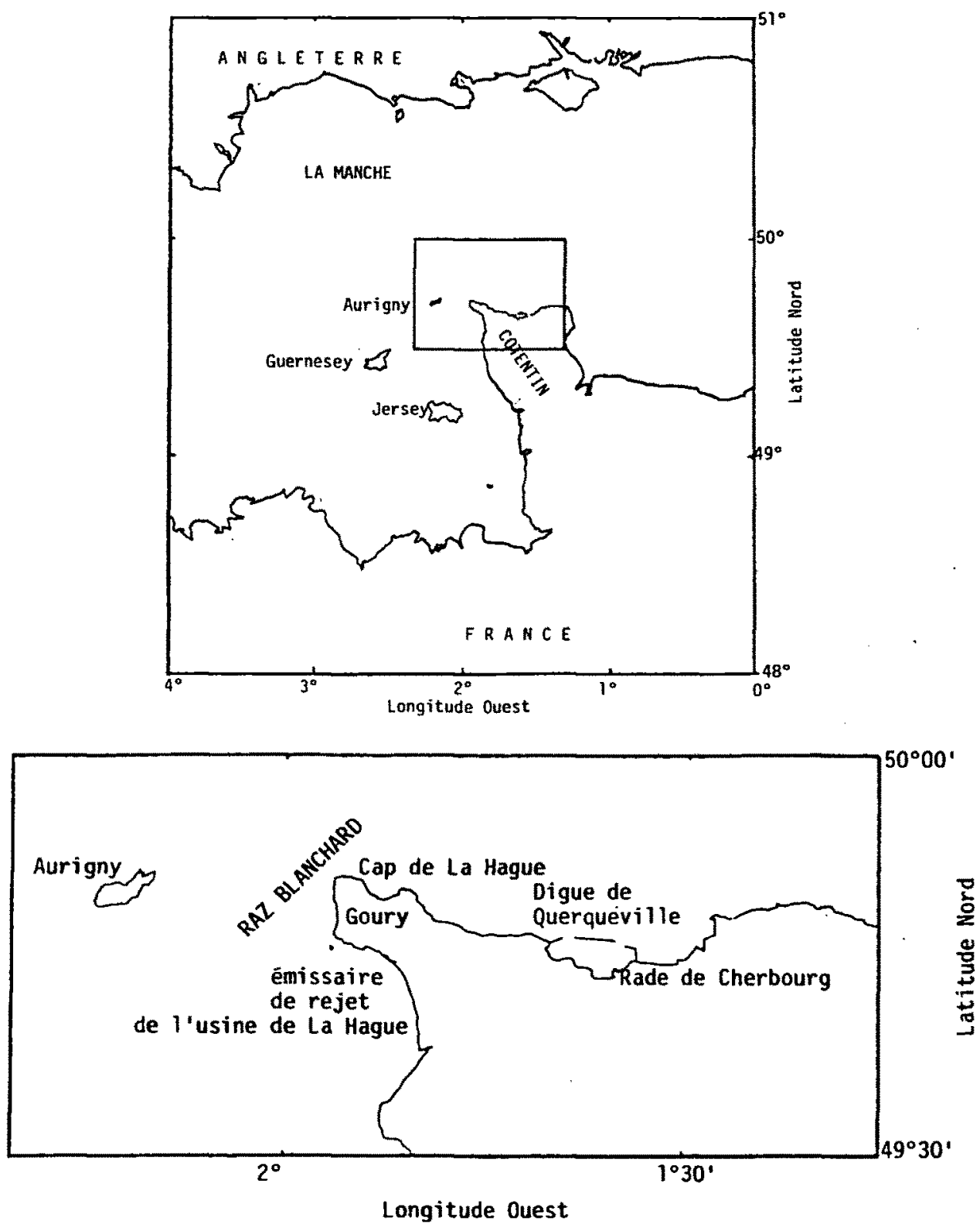

Fig. 1 - Site géographique de l'étude. Location of the study.

$\mathrm{Au}$ niveau toxicologique, les facteurs de concentration(1) de ${ }^{106} \mathrm{Ru}$ sont de 2000 pour les mollusques et les macro-algues, 100 pour les crustacés et 2 pour les poissons [1]. Les coefficients de distribution(2) relatifs aux sédiments peuvent atteindre $10^{4}$ [2]. Ces valeurs ont été obtenues à partir d'eau de mer marquée par ${ }^{106} \mathrm{Ru}$ à l'état de trichlorure de ruthénium. Les auteurs mentionnent que les facteurs de transfert dépendent de la nature physico-chimique du radionucléide.

(1) Les facteurs de concentration sont relatifs à la matière fraîche, ils expriment le quotient de l'activité déterminée par unité de masse dans l'organisme par rapport au volume d'eau de mer correspondant.

(2) Les coefficients de distribution sont relatifs au sédiment sec, ils sont définis selon les critères exprimés dans le cas des facteurs de concentration. 
L'objectif de ce travail est de tracer l'historique de l'évolution temporelle des formes chimiques de ${ }^{106} \mathrm{Ru}$ consécutive aux divers traitements appliqués à l'épuration des effluents, afin de pouvoir interpréter les modalités des transferts du radionucléide dans le milieu marin.

Dans cette optique, nous traiterons successivement les quatre points suivants :

1) un rappel des processus de complexation du ruthénium dans le cycle de retraitement des combustibles irradiés;

2) la description des conditions de rejet des eaux résiduaires de l'usine de La Hague et de l'organisation des études entreprises sur l'environnement marin ;

3) la recherche d'un critère d'évaluation des quantités annuelles de ${ }^{106} \mathrm{Ru}-\mathrm{Rh}$ rejetées en mer permettant d'apprécier l'efficacité des différents procédés d'épuration appliqués au ruthénium des rejets liquides ;

4) le comportement chimique du ${ }^{106} \mathrm{Ru}$ dans les rejets liquides et le milieu marin récepteur; l'accent sera mis sur l'hydrolyse des structures chimiques complexes du radionucléide dans l'eau de mer et ses conséquences dans l'environnement, en utilisant l'algue Fucus serratus comme bioindicateur.

\section{Complexation du ruthénium dans le cycle de retraitement des combustibles irradiés}

Le procédé PUREX utilise le mélange tributylphosphate (TBP) - dodécane comme extractant de l'uranium et du plutonium en milieu nitrique. Le maintien du ruthénium dans la phase aqueuse est une des conditions requises pour séparer efficacement $\mathrm{U}$ et $\mathrm{Pu}$ des produits de fission et d'activation.

La solubilité du ruthénium dans les milieux nitriques est conditionnée par les propriétés oxydantes et complexantes de $\mathrm{HNO}_{3}$ [19]. En présence d'acide concentré $\mathrm{Ru}$ est à l'état de tétroxyde $\left(\mathrm{RuO}_{4}\right)$. Cette forme volatile du ruthénium n'est pas retenue par les systèmes de filtration des effluents gazeux et doit donc être évitée par souci de protection de l'environnement. $\mathrm{RuO}_{4}$ est également instable au contact des tuyaux et enceintes métalliques de l'usine où il est réduit à l'état de $\mathrm{RuO}_{2}$ et constitue alors un danger d'irradiation des personnels travaillant sur le site [18]. Pour éviter ces nuisances, le ruthénium est amené à l'état d'oxydation +3 , dans le but de le maintenir en solution nitrique.

Les complexes du nitrosylruthénium $\left(\mathrm{Ru}(\mathrm{NO})^{3+}\right.$ ) formés à partir des produits de dégradation de l'acide nitrique $[7,25]$ stabilisent $\mathrm{Ru}$ aux états de valence inférieurs et contribuent à sa solubilisation dans la phase aqueuse. La spécificité de l'opération d'extraction U-Pu par la phase TBP - dodécane est cependant susceptible d'être contrariée par la présence de la structure trinitrato, $\mathrm{RuNO}\left(\mathrm{NO}_{3}\right)_{3}\left(\mathrm{H}_{2} \mathrm{O}\right)_{2}$ (Nikol'skii et Shmidt, d'après [3]). La substitution des ligands aquo du complexe trinitrato par deux molécules de TBP aboutit au 
complexe $\mathrm{RuNO}\left(\mathrm{NO}_{3}\right)_{3}(\mathrm{TBP})_{2}$ soluble dans la phase contenant $\mathrm{U}$ et $\mathrm{Pu}$ (Zvyagintsev, d'après [3] et [5]). Selon Sato [25], les complexes du nitrosylruthénium les plus stables en milieux aqueux sont formés par substitution de ligands forts tels que $\mathrm{NO}^{*}, \mathrm{NO}^{+}$et $\mathrm{NO}_{2}{ }^{-}$aux groupements faibles nitrato et aquo. Ces complexes forts sont obtenus au cours des opérations de retraitement des combustibles irradiés usés où l'énergie des réactions chimiques et le rayonnement des radionucléides provoquent la dégradation de l'acide nitrique. Les radicaux libres $\mathrm{NO}^{*}$ neutres sont les plus réactifs. Ils sont générés lors de la radiolyse et jouent un rôle de première importance vis-à-vis du comportement chimique du ruthénium. $\mathrm{NO}^{*}$ cède son électron excédentaire à $\mathrm{Ru}^{3+}$ en s'oxydant à l'état $\mathrm{NO}^{+}$(Fig. 2). Le ruthénium de la structure nitrosylruthénium $\left(\mathrm{Ru}(\mathrm{NO} 3)^{3+}\right)$ est donc réduit à l'état $\mathrm{Ru}^{2+}$.

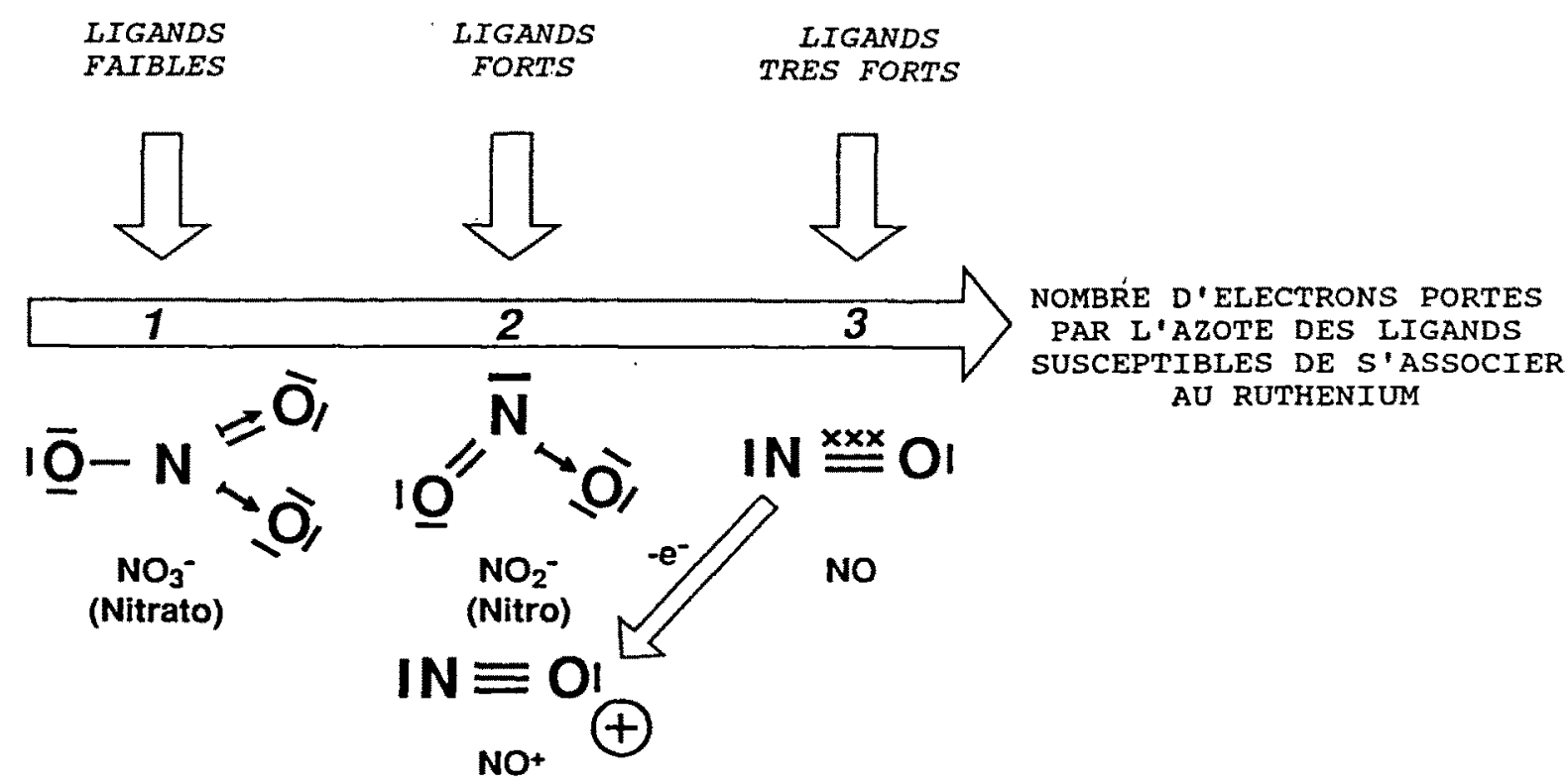

Fig. 2 - Structure électronique des ligands complexants au ráthénium formés dans le procédé Purex, d'après [10].

Electronic structure of the complexing ligands formed in the Purex process, after [10].

L'importance de cette radiolyse et la complexation du ruthénium dépendent de la concentration des produits de fission et d'activation dans la solution nitrique de dissolution des combustibles irradiés. Ainsi, la stabilité des complexes du ruthénium sera liée à la nature du flux neutronique, à son intensité et à la durée d'exposition des combustibles dans les réacteurs. Elle dépendra également de la durée pendant laquelle les combustibles usés seront stockés en piscine de désactivation, avant les opérations de retraitement.

Les effluents les plus sensibles à ce type de complexation par radiolyse nitrique proviennent des ateliers de dissolution des combustibles et du premier cycle d'extraction U-Pu où la teneur en produits de fission et d'activation est la plus forte. 
L'effet de complexation du ruthénium qui contribue à améliorer l'efficacité du procédé de séparation des produits de fission et d'activation perturbe, par contre, les opérations d'épuration des effluents liquides provenant des ateliers de production de l'usine. Le ruthénium présent en phase aqueuse se trouve, en effet, masqué aux réactifs habituellement utilisés pour éliminer l'élément non complexé.

Dans la suite de ce travail, nous mettons l'accent sur les propriétés réactionnelles des produits d'hydrolyse des complexes du nitrosylruthénium vis-à-vis de $\mathrm{MnO}_{2}$ utilisé comme adsorbant et de l'algue Fucus serratus qui est le bioindicateur que nous avons sélectionné dans le milieu marin naturel. Par définition, les produits de l'hydrolyse des complexes du nitrosylruthénium possèdent un ou plusieurs groupements coordinés aquo $\left(\mathrm{H}_{2} \mathrm{O}\right)$ ou hydroxo $\left(\mathrm{OH}^{-}\right)$parmi les six ligands associé à $\mathrm{Ru}(\mathrm{II})$. Nous prendrons ici en considération les produits d'hydrolyse comportant au moins un ligand $\mathrm{OH}^{-}$et symbolisés par $\mathrm{Ru}-\mathrm{OH}$.

\section{Conditions de rejet des eaux résiduaires de l'usine de La Hague et organisation des études sur l'environnement marin}

Les rejets radioactifs liquides de l'usine de La Hague sont constitués des eaux résiduaires obtenues après lavage, rinçage et décontamination des effluents liquides des ateliers de production. Après un contrôle de conformité aux normes de niveau de radioactivité édictées par les autorités françaises, ils sont injectés dans le fort courant marin du Raz Blanchard circulant entre l'île anglo-normande d'Aurigny et le cap de La Hague (Fig. 1). Les créneaux horaires de rejets sont choisis en fonction du cycle de marée, afin d'obtenir une efficacité de dispersion maximale en direction de Manche Est-mer du Nord. Les quantités de ${ }^{106} \mathrm{Ru}-\mathrm{Rh}$ rejetées annuellement depuis la mise en exploitation de l'usine en 1966 sont présentées figure 3.

Des stations d'observation de la radioactivité de l'eau de mer ont été mises en place par l'IPSN et le GEA, en 1974, dans la rade de Cherbourg, à l'extrémité est de la digue de Querqueville et en 1982 à Goury (Fig. 1). Ces deux sites sont situés respectivement à 35 et $5 \mathrm{~km}$ du point de rejet. Les analyses ont été réalisées chaque quinzaine, à partir de prélèvements journaliers de $30 \mathrm{l}$.

De 1975 à 1991, nous avons mesuré chaque mois la radioactivité gamma de l'algue Fucus serratus. Le choix de ce bioindicateur se justifie par son abondance sur l'estran des sites étudiés et son affinité pour ${ }^{106} \mathrm{Ru}$ [11].

Les résultats des mesures de l'environnement sont exprimés sous la forme de moyennes annuelles. Cette présentation fait mieux ressortir l'influence des paramètres physico-chimiques du ruthénium qui sont les critères choisis comme objectifs de ce travail pour interpréter les modalités des transferts de l'élément. Elle permet de limiter l'incidence des variations temporelles de radioactivité imputables à des facteurs climatiques, saisonniers et physiologiques liés au milieu marin naturel lui-même. 
Pour déterminer ${ }^{106} \mathrm{Ru}-\mathrm{Rh}$ dans l'eau de mer, nous avons utilisé la méthode de concentration du ruthénium sur bioxyde de manganèse décrite par Guéguéniat et al. [16]. Elle permet la mesure concomitante de ${ }^{106} \mathbf{R u}-\mathrm{Rh}$ et $125 \mathrm{Sb}$. L'antimoine se dispersant dans l'eau sans participer de façon significative aux processus d'échanges avec les autres constituants du milieu marin, les valeurs de ${ }^{125} \mathrm{Sb}$ donneront une évaluation de l'effet de dilution des rejets en mer $[6,9,17]$. Le tableau I exprime les rapports annuels moyens des activités ${ }^{106} \mathrm{Ru}-\mathrm{Rh} /{ }^{125} \mathrm{Sb}$ rejetées (communication personnelle, Service de prévention et de radioprotection, COGEMA) pendant la période 1974-1991 où le suivi systématique de la radioactivité de l'eau de mer et de l'algue Fucus serratus a été entrepris.

\section{Procédés d'épuration appliqués au ${ }^{106} \mathbf{R u}-\mathrm{Rh}$ des effluents}

Pour obtenir des éléments d'appréciation de l'efficacité des différents procédés d'épuration appliqués aux effluents, il nous est apparu nécessaire de ne pas limiter notre approche à la prise en compte des seules valeurs brutes d'activités annuelles rejetées (Fig. 3). Nous avons cherché à exprimer les rejets annuels par rapport aux quantités de combustibles retraités. Cette évaluation tient compte des trois types de combustibles pris en charge par l'usine de La Hague de 1966 à 1992 :

- la filière "uranium naturel-graphite gaz", de 1966 à 1987 ;

- la filière "eau légère" qui, à partir de 1976, a constitué l'essentiel du tonnage retraité ;

- la filière "neutrons rapides", de 1979 à 1984.

\section{TABLEAU I}

Bilan des quotients d'activités moyennes annuelles de ${ }^{106} \mathrm{Ru}-\mathrm{Rh} / 125 \mathrm{Sb}(R)$ évalués à partir des rejets de l'usine de La Hague de 1974 à 1991 ${ }^{106} \mathrm{Ru}-\mathrm{Rh} /{ }^{125} \mathrm{Sb}$ annual average activity ratios estimated from La Hague releases, 1974-1991

\begin{tabular}{|c|c|c|c|c|c|c|c|}
\hline \multicolumn{2}{|c|}{${ }_{106} \mathrm{Ru}-\mathrm{Rh} / 125 \mathrm{Sb}$} & \multicolumn{2}{|c|}{${ }^{106} \mathrm{Ru}-\mathrm{Rh} / 125 \mathrm{Sb}$} & \multicolumn{2}{|c|}{${ }^{106} \mathrm{Ru}-\mathrm{Rh} / 125 \mathrm{Sb}$} & \multicolumn{2}{|c|}{${ }^{106} \mathrm{Ru}-\mathrm{Rh} / 125 \mathrm{Sb}$} \\
\hline Année & $\boldsymbol{R}$ & Année & $\boldsymbol{R}$ & Année & $R$ & Année & $R$ \\
\hline 1974 & 7,8 & 1979 & 13,9 & 1984 & 5,3 & 1989 & 6,5 \\
\hline 1975 & 11,5 & 1980 & 15,2 & 1985 & 8,1 & 1990 & 10,0 \\
\hline 1976 & 15,4 & 1981 & 15,6 & 1986 & 5,7 & 1991 & 1,7 \\
\hline 1977 & 8,1 & 1982 & 11,7 & 1987 & 5,8 & & \\
\hline 1978 & 12,9 & 1983 & 4,5 & 1988 & 5,2 & & \\
\hline
\end{tabular}




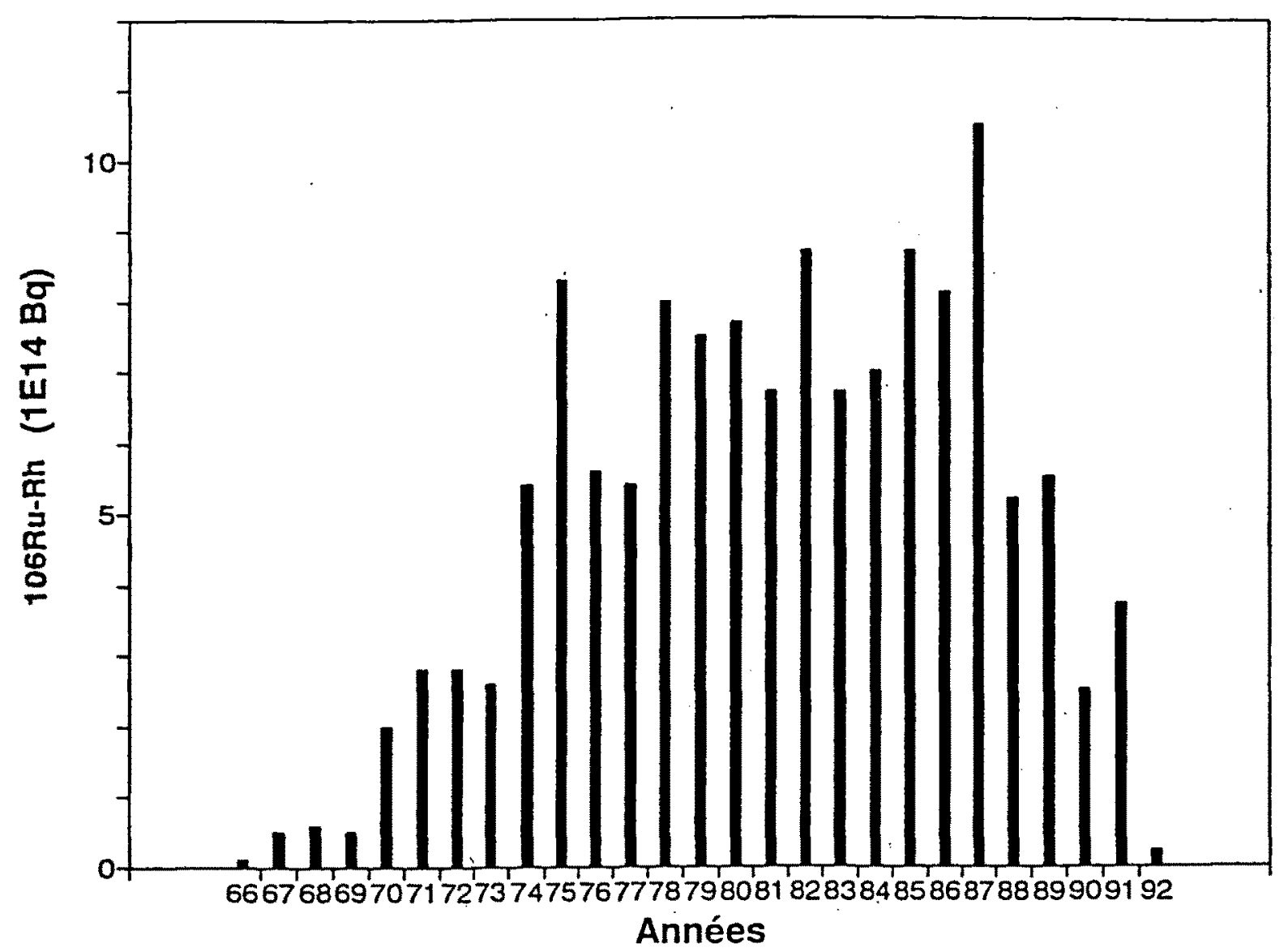

Fig. 3 - Bilan des rejets annuels de ${ }^{106}$ Ru-Rh effectués par l'usine de La Hague, entre 1966 et 1992.

${ }^{106}$ Ru-Rh annuel releases from the La Hague reprocessing plant, 1966-1992.

\subsection{Evaluation comparative des rejets annuels de ${ }^{106} \mathbf{R u}-\mathrm{Rh}:$ normalisation des quantités rejetées}

Nous avons substitué à la notion de tonnage de combustible celle d'équivalent ruthénium qui prend en considération la masse de tous les isotopes du ruthénium entrant dans le cycle de retraitement. Le tableau II indique la masse de ruthénium retraité et le détail des contributions de chaque type de combustible.

Dans cette perspective et pour les besoins de ce travail, nous considérerons l'usine de La Hague comme une unité de séparation du ruthénium. Nous utiliserons le concept de "rejet annuel normalisé" qui définit l'activité en ${ }^{106} \mathrm{Ru}-\mathrm{Rh}$, exprimée en térabecquerel $(\mathrm{TBq})$, rejetée annuellement dans le milieu marin, pour $1 \mathrm{~kg}$ de ruthénium pris en charge.

\subsection{Evolution temporelle des rejets annuels normalisés}

La figure 4 exprime l'évolution des rejets annuels normalisés de ${ }^{106} \mathrm{Ru}-\mathrm{Rh}$ pour la période 1966-1992. Pour une année considérée, le traitement employé pour l'élimination du ruthénium des effluents est d'autant plus efficace que la 
valeur du rejet annuel normalisé est faible. De 1966 à 1975, on observe une tendance générale à une augmentation des rejets annuels normalisés. La valeur maximale de 1975 correspond à $9,6 \mathrm{TBq}$ de ${ }^{106} \mathrm{Ru}-\mathrm{Rh}$ rejetés par kilogramme de ruthénium retraité. A partir de 1976, l'efficacité du procédé a considérablement progressé par rapport à 1975 : les rejets normalisés ont été divisés par 7,4 en 1983, par 16 en 1989 et par 600 en 1992.

\section{TABLEAU II}

Bilan des masses de ruthénium (tous isotopes confondus) retraitées par I'usine de La Hague entre 1966 et 1992 (4). Le détail des contributions de chacun des types de combustibles est donné en (1), (2) et (3) Ruthenium masses (all isotopes) reprocessed, 1966-1992

\begin{tabular}{|c|c|c|c|c|}
\hline$y_{*}$ & $\begin{array}{c}\text { Combustibles } \\
\text { uranium naturel } \\
\text { graphite gaz } \\
\text { (en kg) } \\
(1)\end{array}$ & $\begin{array}{c}\text { Combustibles } \\
\text { eau ordinaire } \\
\text { (en kg) } \\
\text { (2) }\end{array}$ & $\begin{array}{c}\text { Combustibles } \\
\text { neutrons rapides } \\
\text { (en kg) } \\
(\mathbf{3}) \\
\end{array}$ & $\begin{array}{l}\text { Masse } \\
\text { de ruthénium } \\
\text { retraite } \\
\text { (en kg) } \\
\text { (4) }\end{array}$ \\
\hline 1966 & 24 & & & 24 \\
\hline 1967 & 24 & & & 24 \\
\hline 1968 & 37 & & & 37 \\
\hline 1969 & 50 & & & 50 \\
\hline 1970 & 40 & & & 40 \\
\hline 1971 & 47 & & & 47 \\
\hline 1972 & 64 & & & 64 \\
\hline 1973 & 94 & & & 94 \\
\hline 1974 & 71 & & & 71 \\
\hline 1975 & 87 & & & 87 \\
\hline 1976 & 44 & 46 & & 90 \\
\hline 1977 & 71 & 46 & & 117 \\
\hline 1978 & 76 & 77 & & 153 \\
\hline 1979 & 31 & 169 & 12 & 212 \\
\hline 1980 & 34 & 231 & 8 & 272 \\
\hline 1981 & 13 & 200 & 10 & 223 \\
\hline 1982 & 45 & 338 & & 383 \\
\hline 1983 & 5 & 453 & 17 & 525 \\
\hline 1984 & 13 & 530 & 17 & 560 \\
\hline 1985 & 23 & 722 & & 745 \\
\hline 1986 & 11 & 699 & & 710 \\
\hline 1987 & 15 & 898 & & 913 \\
\hline 1988 & & 730 & & 730 \\
\hline 1989 & & 973 & & 973 \\
\hline 1990 & & 1108 & & 1108 \\
\hline 1991 & & 1397 & & 1397 \\
\hline 1992 & & 1469 & & 1469 \\
\hline
\end{tabular}




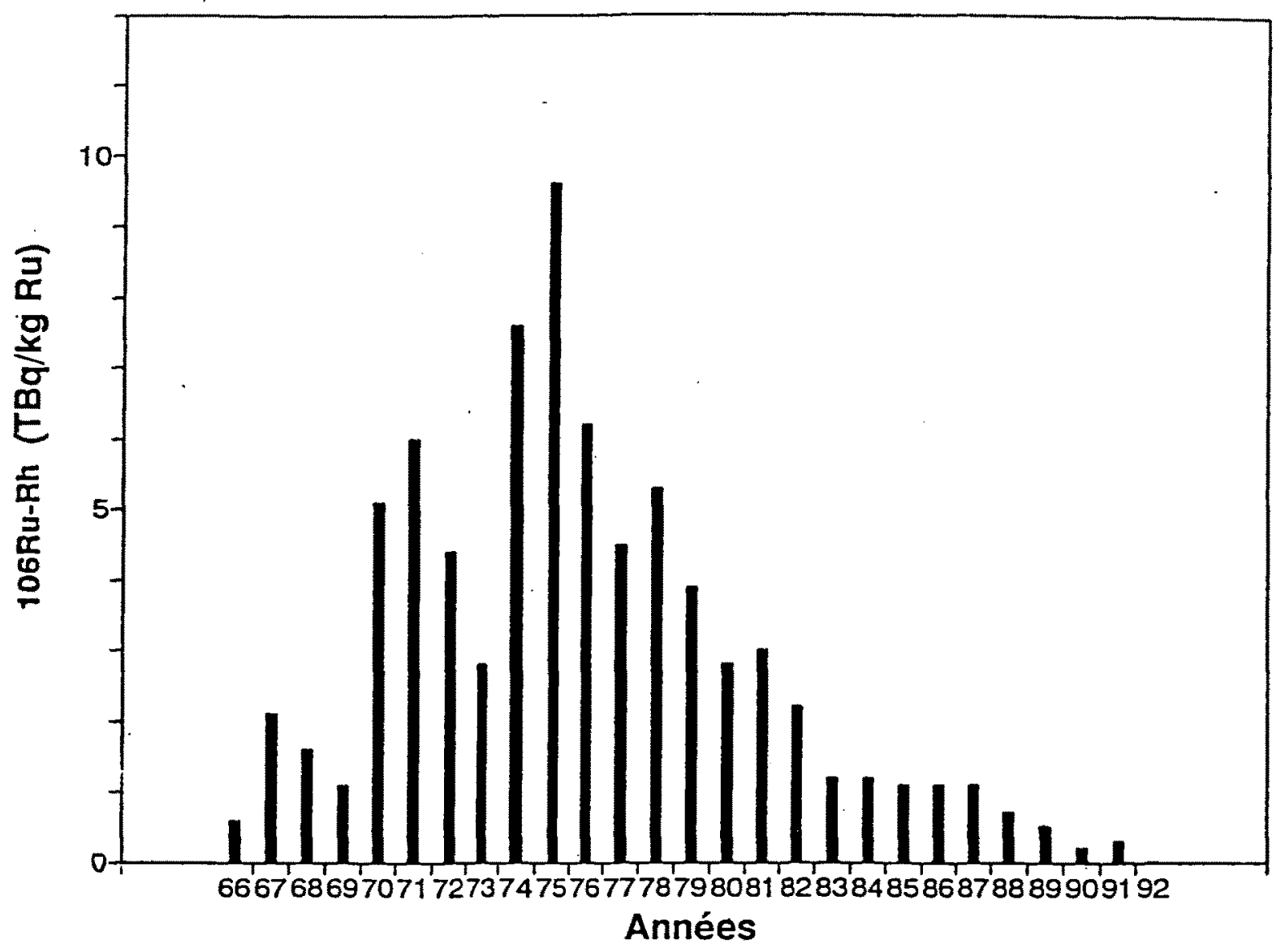

Fig. 4 - Bilan des rejets annuels normalisés de ${ }^{106}$ Ru-Rh effectués par l'usine de La Hague, entre 1966 et 1992.

${ }^{106} \mathrm{Ru}-\mathrm{Rh}$ normalised annual releases from the La Hague reprocessing plant, 1966-1992.

\subsection{Description et commentaires sur les procédés d'épuration appliqués au ruthénium des effluents, de 1966 à 1991}

La décision de mettre en œuvre un procédé de traitement des effluents plus particulièrement axé sur ${ }^{106} \mathrm{Ru}$ a été prise en 1967. Au cours de la période 1968-1971, les procédés de coprécipitation par les oxydes de fer appliqués à l'épuration des eaux potables urbaines ont été adaptés au cas du ruthénium des effluents radioactifs sans permettre l'élimination de ses complexes stables. C'est en 1971 qu'un procédé plus efficace d'entraînement de ${ }^{106} \mathrm{Ru}$ par le sulfure de cobalt en milieu basique a été mis au point [4]. L'adaptation de la technique aux conditions industrielles d'exploitation a nécessité plusieurs années. Les modalités d'introduction des réactifs dans les cuves de réaction ont été améliorées en 1973 et, à partir de 1975, l'accent a été mis sur le contrôle du pH qui fut le paramètre déterminant. L'utilisation de solutions de soude diluée et de pompes doseuses a, tout d'abord, permis de réaliser l'homogénéité nécessaire à l'intérieur des cuves de réaction et, en 1982, la fiabilité des mesures de $\mathrm{pH}$ a été obtenue. La maîtrise des opérations d'épuration de ${ }^{106} \mathrm{Ru}$ par le sulfure de cobalt par "voie humide" date de 1983 à la station STE2 avec la mise en service d'automates pour gérer la distribution des réactifs et le contrôle du 
pH. Dès sa mise en service en 1990, la station STE3 de traitement des effluents liquides a bénéficié de cette technologie. Ces améliorations ont été accompagnées de mesures pour limiter la concentration des ions nitrites dans les effluents. La progression constatée en 1988 est attribuable à la dilution des effluents de la chaîne principale par des effluents de faible activité, contenant peu d'ions nitrites complexants.

Le procédé d'épuration a ainsi fonctionné jusqu'en 1989. Il a permis de supporter l'augmentation de la charge de production. Celle-ci, inférieure à $100 \mathrm{~kg}$ équivalent ruthénium avant 1977, était proche de $1000 \mathrm{~kg}$ en 1989 (Tab. II). A compter de 1988-1989, dans le cadre d'une politique de réduction du niveau d'activité des rejets, les effluents provenant de l'atelier de dissolution des combustibles et du premier cycle d'extraction U-Pu ont été traités séparément du procédé "voie humide" pour être évaporés à sec et vitrifiés. Ce procédé, qualifié de "voie sèche" fonctionne de façon industrielle depuis 1990. Le problème de l'élimination des formes complexes du ruthénium inaccessibles au procédé d'épuration utilisant le sulfure de cobalt se trouve ainsi résolu. En 1992 , cette technologie a permis une productivité de près de $1500 \mathrm{~kg}$ équivalent ruthénium (Tab. II) pour un rejet annuel limité à $23 \mathrm{TBq}$ de ${ }^{106} \mathrm{Ru}-\mathrm{Rh}$. Le rejet annuel normalisé de 1992 a été de $0,016 \mathrm{TBq}$, il constitue le meilleur bilan enregistré depuis 1966 (Fig. 4).

\section{Comportement chimique du ruthénium dans les rejets liquides de l'usine de La Hague et dans le milieu marin récepteur}

\subsection{Résultats expérimentaux}

Les capacités d'entraînement de la méthode utilisant le dioxyde de manganèse IV [15] ont été testées au laboratoire, par rapport au ${ }^{106} \mathrm{Ru}$ contenu dans les rejets de l'usine de La Hague, au cours de la période 1966-1991 (Tab. III).

\section{TABLEAU III}

Bilan des tests de laboratoire réalisés entre 1966 et 1991 permettant d'établir les rendements d'extraction de ${ }^{106} \mathrm{Ru}$ par le bioxyde de manganèse à pH 8,6, dans une eau de mer marquée par des rejets

Laboratory tests (1966-1991) and ${ }^{106} \mathrm{Ru}$ extraction yields by $\mathrm{MnO}_{2}$ (pH 8.6), in sea water labelled by releases

$\begin{array}{ll}1966-1968 & \text { Rendement d'extraction }(\%)=100 \\ 1972-1973 & 40<\text { Rendements d'extraction }(\%)<65 \\ 1984-1985 & 16<\text { Rendements d'extraction }(\%)<28 \\ 1988-1989 & 0<\text { Rendements d'extraction }(\%)<20 \\ 1990-1991 & 30<\text { Rendements d'extraction }(\%)<60\end{array}$


Les concentrations de ${ }^{125} \mathrm{Sb}$ et ${ }^{106} \mathrm{Ru}-\mathrm{Rh}$ dans les eaux de la rade de Cherbourg et à Goury ( $\mathrm{Tab}$. IV et $\mathrm{V}$ ) correspondent aux moyennes annuelles des 24 mesures bi-hebdomadaires réalisées de 1974 à 1991.

Le tableau VI fait le bilan de l'activité de ${ }^{106} \mathrm{Ru}-\mathrm{Rh}$ dans l'algue Fucus serratus en rade de Cherbourg et à Goury. Chacune des valeurs correspond à la moyenne des 12 mesures mensuelles réalisées de 1975 à 1991.

TABLEAU IV

Bilan des activités moyennes annuelles de ${ }^{125} \mathrm{Sb}$ dans l'eau de mer, à l'entrée ouest de la rade de Cherbourg, entre 1974 et 1991 et à Goury, entre 1982 et 1991

$125 \mathrm{Sb}$ annual average activities in sea water, Cherbourg harbour west entry, 1974-1991, and Goury, 1982-1991

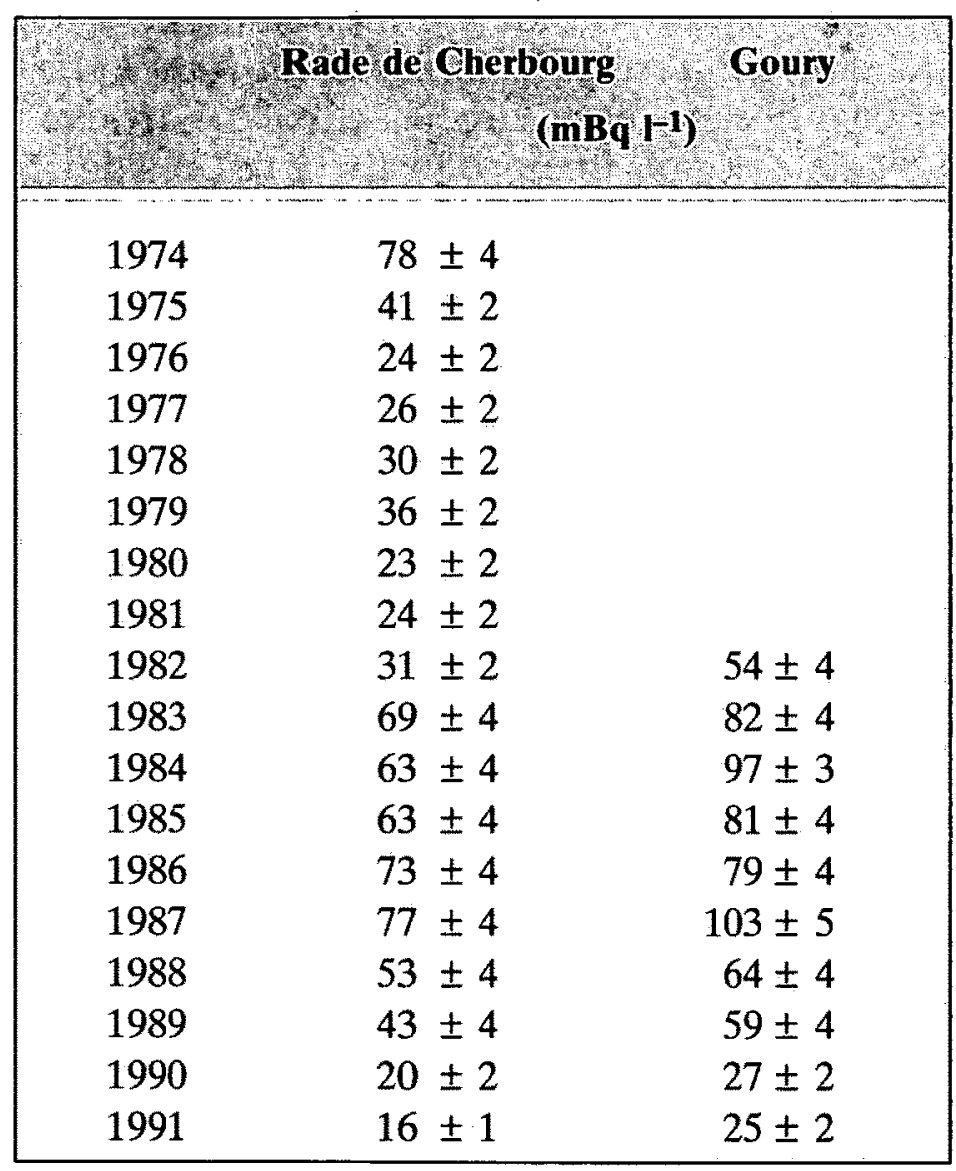

4.2 Hydrolyse des structures chimiques complexes de ${ }^{106} \mathrm{Ru}$ dans les rejets liquides et l'eau de mer du milieu naturel

a) Mise en évidence de l'hydrolyse des complexes du nitrosylruthénium : signification des résultats obtenus avec le dioxyde de manganèse IV comme adsorbant

Le mode de préparation de l'adsorbant (eau de mer à $\mathrm{pH} 8,2$ et réduction de l'ion permanganate à la concentration $1,6 \times 10^{-3} \mathrm{M}$ par l'eau oxygénée) implique un $\mathrm{pH}$ du milieu proche de 8,6. Dans ces conditions, la charge élec- 
TABLEAU $\mathrm{V}$

Bilan des activités moyennes annuelles de ${ }^{106} \mathbf{R u}-\mathbf{R h}$ mesurées sur le bioxyde de manganèse à pH 8,6, dans l'eau de mer prélevée à l'entrée ouest de la rade de Cherbourg, entre 1974 et 1991 et à Goury, pendant la période 1982-1991 ${ }^{106} \mathrm{Ru}-\mathrm{Rh}$ annual average activities measured on $\mathrm{MnO}_{2}$ (pH 8.6) in sea water, Cherbourg harbour west entry, 1974-1991, and Goury, 1982-1991

\begin{tabular}{|ccc|}
\hline & Rade de Cherbourg & Goury \\
& & \\
(mBq 1 1)
\end{tabular}

trique négative portée par $\mathrm{MnO}_{2}$ est favorable à une adsorption de type cationique [20-21]. Le processus d'entraînement de ${ }^{106} \mathrm{Ru}$, qui est à plus de $97 \%$ sous formes anioniques dans l'eau de mer $[10,15]$ s'inclue dans le cadre général d'interaction des oxyhydroxydes adsorbants avec les éléments hydrolysables [8]. Il fait intervenir les ligands hydroxo des complexes du nitrosylruthénium $(\mathrm{Ru}-\mathrm{OH})$ hydrolysés, précédemment définis, et ceux de l'adsorbant $(\mathrm{Mn}-\mathrm{OH})$ :

$$
\mathrm{Ru}-\mathrm{OH}+\mathrm{HO}-\mathrm{Mn} \rightarrow \mathrm{Ru}-\mathrm{O}-\mathrm{Mn}+\mathrm{H}_{2} \mathrm{O}
$$

Les résultats des tests d'entraînement de ${ }^{106} \mathrm{Ru}$ par $\mathrm{MnO}_{2}$ pratiqués à partir des rejets (Tab. III) font apparaître que les rendements de fixation de $100 \%$ obtenus au cours des premières années d'exploitation de l'usine ont progressivement diminué lorsque l'optimisation du procédé d'épuration par "voie humide" a été obtenue. En 1988-1989, la technique de concentration sur $\mathrm{MnO}_{2}$ a montré son inefficacité vis-à-vis de rejets où la concentration en ${ }^{106} \mathrm{Ru}-\mathrm{Rh}$ atteignait le niveau de $10^{6} \mathrm{~Bq}^{-1}$. La méthode de préparation de l'adsorbant 
TABLEAU VI

Bilan des activités moyennes annuelles de $106 \mathrm{Ru}-\mathrm{Rh}$ mesurées dans l'algue Fucus serratus dans le secteur ouest de la rade de Cherbourg et à Goury, entre 1975 et 1991

106 Ru-Rh annual average activities measured in Fucus serratus in the western part of Cherbourg harbour and at Goury

\begin{tabular}{|c|c|c|}
\hline$\cdots$ & \multicolumn{2}{|c|}{ 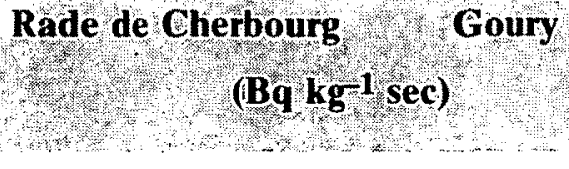 } \\
\hline 1975 & $625 \pm 30$ & $1125 \pm 55$ \\
\hline 1976 & $275 \pm 25$ & $605 \pm 30$ \\
\hline 1977 & $330 \pm 35$ & $730 \pm 35$ \\
\hline 1978 & $285 \pm 30$ & $775 \pm 40$ \\
\hline 1979 & $515 \pm 25$ & $855 \pm 45$ \\
\hline 1980 & $260 \pm 25$ & $1395 \pm 70$ \\
\hline 1981 & $485 \pm 25$ & $1910 \pm 95$ \\
\hline 1982 & $780 \pm 40$ & $1065 \pm 55$ \\
\hline 1983 & $515 \pm 25$ & $1320 \pm 65$ \\
\hline 1984 & $360 \pm 35$ & $1020 \pm 50$ \\
\hline 1985 & $315 \pm 30$ & $570 \pm 30$ \\
\hline 1986 & $390 \pm 40$ & $660 \pm 35$ \\
\hline 1987 & $275 \pm 25$ & $405 \pm 40$ \\
\hline 1988 & $200 \pm 20$ & $390 \pm 40$ \\
\hline 1989 & $135 \pm 30$ & $310 \pm 30$ \\
\hline 1990 & $190 \pm 20$ & $130 \pm 20$ \\
\hline 1991 & $30 \pm 5$ & $25 \pm 5$ \\
\hline
\end{tabular}

n'ayant pas été modifiée entre 1966 et 1989 , nous considérons que le caractère chimique du ruthénium dans les rejets les plus marqués par ${ }^{106} \mathrm{Ru}$ était celui de structures non hydrolysées dans les conditions d'expérience (eau de mer à $\mathrm{pH}$ 8,6). A partir de 1990-1991, les performances de la technique $\mathrm{MnO}_{2}$ appliquée au ${ }^{106} \mathrm{Ru}$ de ces rejets liquides se sont améliorées à la suite du traitement par "voie sèche" des effluents les plus riches en complexes forts, difficilement hydrolysables.

L'utilisation du dioxyde de manganèse IV adsorbant comme moyen d'évaluer l'hydrolyse des formes chimiques complexes du ruthénium dans les rejets liquides est également applicable à l'eau de mer du milieu naturel. Le tableau VII donne les rapports des moyennes annuelles déterminées dans l'eau de mer à Cherbourg par rapport à Goury pour ${ }^{125} \mathrm{Sb}$ et ${ }^{106} \mathrm{Ru}-\mathrm{Rh}$, entre 1982 et 1991 . Les quotients de concentration relatifs à ${ }^{125} \mathrm{Sb}$ (colonne 1) expriment la dilution par l'eau de mer intervenue entre les deux sites. Les valeurs enregistrées sont inférieures à l'unité, elles révèlent un effet de dilution plus important à Cherbourg qu'à Goury. Ce phénomène de dilution par l'eau de mer dépend des conditions hydrodynamiques du milieu naturel [6]. Il est plus marqué en 
1982, 1984 et 1991 . Le bilan relatif à $106 \mathrm{Ru}-\mathrm{Rh}$ (Tab. VII, colonne 2) montre que l'effet de dilution constaté avec ${ }^{125} \mathrm{Sb}$ se trouve globalement confirmé avec ${ }_{106} \mathrm{Ru}$ pendant la période 1982-1989. Au cours du transit entre Goury et Cherbourg, ${ }^{106} \mathrm{Ru}$ se mélange donc avec les masses d'eaux sans montrer d'évolution notable du caractère inerte des complexes du nitrosylruthénium. A partir de 1990 , on constate une première tendance des complexes de ${ }^{106} \mathrm{Ru}$ à évoluer vers des formes chimiques plus réactives vis-à-vis de $\mathrm{MnO}_{2}$. C'est cependant, en 1991 que cette modification du comportement de ${ }^{106} \mathrm{Ru}$ de l'eau de mer est mieux observée. La valeur ${ }^{106} \mathrm{Ru}-\mathrm{Rh}$ est 1,46 fois plus élevée à Cherbourg qu'à Goury, malgré un effet de dilution plus important à Cherbourg. On peut donc considérer que l'hydrolyse des complexes du ruthénium est à l'origine de l'augmentation des capacités de fixation de ${ }^{106} \mathrm{Ru}$ sur $\mathrm{MnO}_{2}$.

TABLEAU VII

Bilan des quotients d'activités moyennes annuelles évalués, dans l'eau de mer, à I'entrée ouest de la rade de Cherbourg par rapport à Goury pour ${ }^{125} \mathrm{Sb}$ (1) et ${ }^{106} \mathbf{R u}-\mathbf{R h}$ mes (2), entre 1982 et 1991

$125 \mathrm{Sb}$ (1) and ${ }^{106} \mathrm{Ru}-\mathrm{Rh}$ meas (2) annual average activity ratios in sea water, Cherbourg harbour west entry vs Goury, 1982-1991

\begin{tabular}{|ccc|}
\hline & 125Sb à Cherbourg & 106Ru-Rh à Cherbourg \\
\cline { 2 - 3 } & 125Sb à Goury & 106Ru-Rh à Goury \\
& $\mathbf{( 1 )}$ & $\mathbf{( 2 )}$ \\
1982 & & 0,52 \\
1983 & 0,58 & 0,74 \\
1984 & 0,84 & 0,66 \\
1985 & 0,64 & 0,83 \\
1986 & 0,78 & 0,82 \\
1987 & 0,92 & 0,73 \\
1988 & 0,75 & 0,93 \\
1989 & 0,82 & 0,61 \\
1990 & 0,73 & 0,91 \\
1991 & 0,74 & 1,46 \\
\hline
\end{tabular}

b) Evaluation de la vitesse d'hydrolyse des complexes du ruthénium, à partir du déficit de l'eau de mer en $106 R u-R h$

Il est possible de quantifier la fraction du ruthénium, à l'état de complexes stables dans l'eau de mer, qui échappe à l'adsorption sur le dioxyde de manganèse IV. Le rapport $R$ des activités annuelles ${ }^{106} \mathrm{Ru}-\mathrm{Rh} /{ }^{125} \mathrm{Sb}$ dans les rejets liquides de l'usine de La Hague (Tab. I) permet de calculer, à partir de la valeur de ${ }^{125} \mathrm{Sb}$ déterminée dans l'eau de mer ([125 Sb]), la quantité annuelle théorique de ${ }^{106} \mathrm{Ru}-\mathrm{Rh}\left({ }^{106} \mathrm{Ru}-\mathrm{Rh}_{\mathrm{th}}\right)$ apportée par les rejets (dans l'hypothèse où ${ }^{106} \mathrm{Ru}$ et ${ }^{125} \mathrm{Sb}$ ont le même comportement) :

$$
{ }^{106} \mathrm{Ru}-\mathrm{Rh}_{\mathrm{th}}=\left[{ }^{125} \mathrm{Sb}\right] \times R
$$


La différence entre ${ }^{106} \mathrm{Ru}-\mathrm{Rh}_{\text {th }}$ et la valeur de ${ }^{106} \mathrm{Ru}-\mathrm{Rh}$ déterminée sur $\mathrm{MnO}_{2}$ caractérise le "déficit" de la mesure de ${ }^{106} \mathrm{Ru}-\mathrm{Rh}$ dans l'eau de mer (Tab. V). Les "déficits annuels moyens" sont exprimés tableau VIII pour les stations de la rade de Cherbourg et Goury, en \% par rapport à $106 \mathrm{Ru}-\mathrm{Rh}_{\mathrm{th}}$. Les résultats tiennent compte d'un retard d'impact des rejets à Cherbourg de 1 à 3 semaines par rapport à la manifestation décelée à Goury [6, 22-24]. Ce retard restreint par rapport aux périodes radioactives de ${ }^{125} \mathrm{Sb}(2,7$ ans $)$ et de ${ }^{106} \mathrm{Ru}(1 \mathrm{an})$ permet de négliger dans les calculs l'incidence de la décroissance radioactive des éléments.

TABLEAU VIII

Bilan des déficits annuels moyens de I'eau de mer en ${ }^{106} \mathbf{R u}$-Rh calculés à partir des valeurs obtenues en utilisant la méthode de concentration du ruthénium sur $\mathrm{MnO}_{2}$. Les résultats sont exprimés en \% par rapport à ${ }^{106} \mathbf{R} u-R h_{\text {th }}$ apporté par les rejets dans les eaux de la rade de Cherbourg (de 1974 à 1991) et de Goury (de 1982 à 1991)

Sea water annual deficits in $106 \mathrm{Ru}-\mathrm{Rh}$ estimated using $\mathrm{Ru}$ concentration on $\mathrm{MnO}_{2}$ vs ${ }^{106} \mathrm{Ru}-\mathrm{Rh}_{\text {th }}$ brought by the releases in Cherbourg harbour, 1974-1991, and at Goury, 1982-1991

\begin{tabular}{|c|c|c|}
\hline & Rade de Cherbourg & Goury \\
\hline & $(\%)$ & \\
\hline 1974 & $53 \pm 42$ & \\
\hline 1975 & $41 \pm 20$ & \\
\hline 1976 & $60 \pm 24$ & \\
\hline 1977 & $23 \pm 23$ & \\
\hline 1978 & $57 \pm 26$ & \\
\hline 1979 & $58 \pm 17$ & \\
\hline 1980 & $69 \pm 17$ & \\
\hline 1981 & $59 \pm 26$ & \\
\hline 1982 & $50 \pm 30$ & $47 \pm 33$ \\
\hline 1983 & $42 \pm 28$ & $34 \pm 21$ \\
\hline 1984 & $53 \pm 27$ & $54 \pm 19$ \\
\hline 1985 & $64 \pm 30$ & $66 \pm 19$ \\
\hline 1986 & $54 \pm 30$ & $49 \pm 20$ \\
\hline 1987 & $67 \pm 28$ & $66 \pm 18$ \\
\hline 1988 & $53 \pm 24$ & $59 \pm 24$ \\
\hline 1989 & $70 \pm 14$ & $64 \pm 26$ \\
\hline 1990 & $45 \pm 15$ & $56 \pm 19$ \\
\hline 1991 & $12 \pm 5$ & $60 \pm 9$ \\
\hline
\end{tabular}

La figure 5 exprime l'évolution de cette fraction de ${ }^{106} \mathrm{Ru}-\mathrm{Rh}$ déficitaire à Cherbourg et Goury, en fonction de la masse de ruthénium entrant dans l'usine de La Hague. Avant 1982, les déficits en ${ }^{106} \mathrm{Ru}-\mathrm{Rh}$ à Cherbourg sont généra- 
lement compris entre $41 \%$ et $60 \%$. L'année 1977 enregistre une valeur minimale de $23 \%$ et l'année 1980 un maximum de $69 \%$. Au cours de la période 1982-1989 où les mesures ont été réalisées conjointement dans les deux stations, les déficits augmentent en fonction de la quantité de ruthénium prise en charge. Les écarts entre les valeurs enregistrées à Goury et à Cherbourg sont inférieurs à $8 \%$. L'évolution chimique du ${ }^{106} \mathrm{Ru}$, au cours de son transit entre les deux stations, est donc peu marquée. Le comportement de ${ }^{106} \mathrm{Ru}$ confirme le caractère complexe évoqué à partir des mesures du radionucléide obtenues dans l'eau de mer, avec la méthode utilisant $\mathrm{MnO}_{2}$ comme adsorbant. A partir des années 1990-1991, une diminution générale du déficit en ruthénium est observée à chacune des stations, et plus particulièrement à Cherbourg. Cette situation est attribuable à l'hydrolyse des complexes de ${ }^{106} \mathrm{Ru}$ qui intervient au cours du transit des masses d'eaux entre l'émissaire et Goury d'une part, entre Goury et Cherbourg d'autre part. Pour l'année 1991, le résultat moyen des observations réalisées sur le site de la rade de Cherbourg est particulièrement révélateur. Près de $50 \%$ du ${ }^{106} \mathrm{Ru}$ de l'eau de mer non adsorbé sur $\mathrm{MnO}_{2}$ à Goury évolue, au cours du transit des eaux vers la rade de Cherbourg, en donnant des structures chimiques hydrolysées adsorbables.

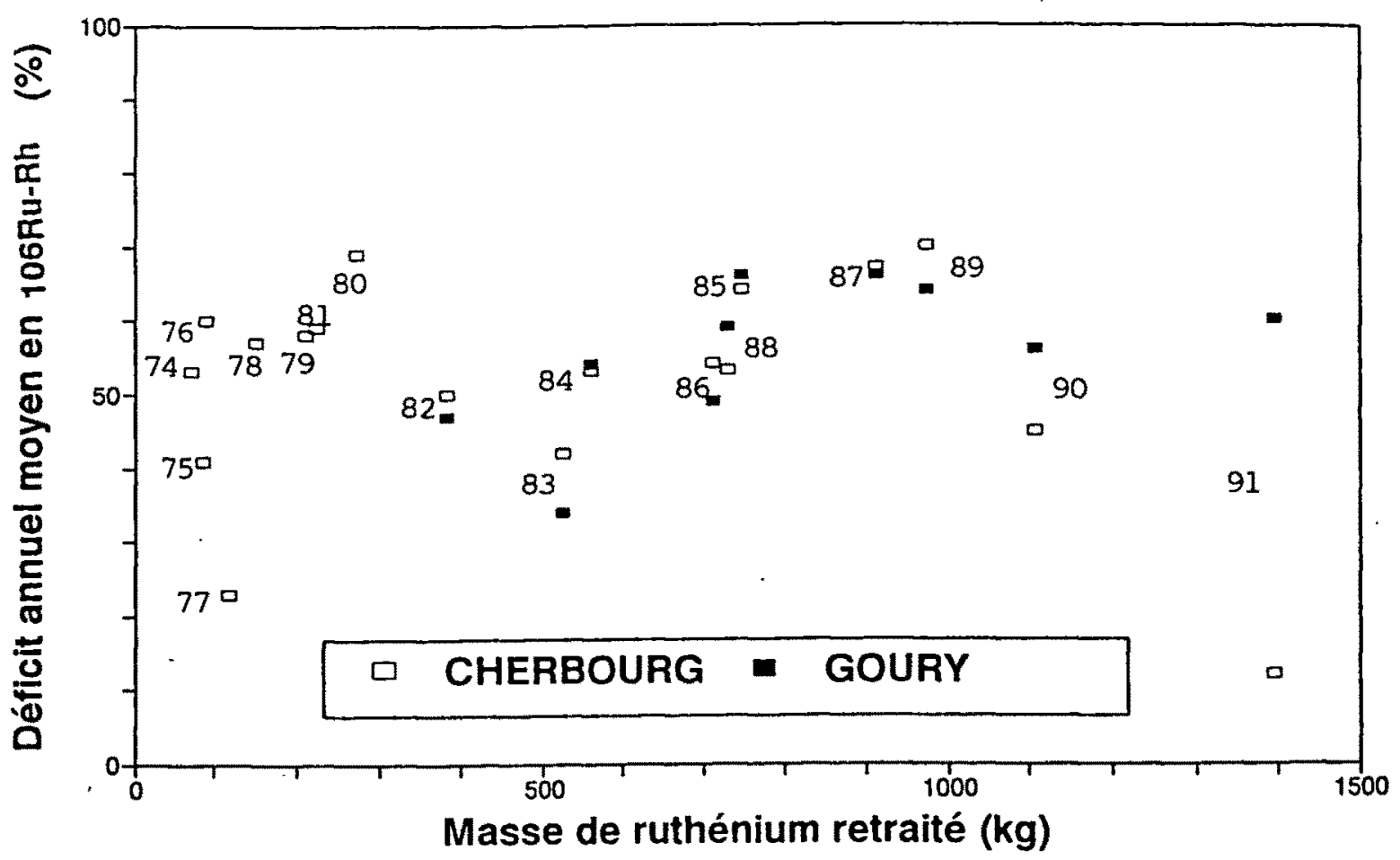

Fig. 5 - Bilan des déficits moyens annuels de ${ }^{106}$ Ru-Rh évalués dans l'eau de mer, à l'entrée ouest de la rade de Cherbourg entre 1974 (74) et 1991 (91) et à Goury entre 1982 (82) et 1991 (91), exprimés en fonction de la masse de ruthénium retraité.

${ }^{106}$ Ru-Rh annual deficits estimated in sea water vs $R u$ masses reprocessed, Cherboug harbour west entry, 1974-1991, and Goury, 1982-1991. 


\subsection{Biodisponibilité de ${ }^{106}$ Ru par rapport à l'algue Fucus serratus}

Le tableau IX, établi à partir des valeurs de ${ }^{106} \mathrm{Ru}-\mathrm{Rh}$ mesurées dans Fucus serratus (Tab. VI) et des activités de ${ }^{106} \mathrm{Ru}-\mathrm{Rh}$ rejetées annuellement (Fig. 3), permet d'évaluer l'impact des rejets de ruthénium sur la radioactivité de l'algue dans la rade de Cherbourg et à Goury entre 1975 et 1991. La valeur de l'impact exprime l'activité en bequerel d'1 kg d'algue à l'état $\mathrm{sec}$, pour $1 \mathrm{GBq}$ de ${ }^{106} \mathrm{Ru}-\mathrm{Rh}$ rejeté en mer.

La disparité constatée entre les différentes valeurs d'impact de ${ }^{106} \mathbf{R u}-\mathbf{R h}$ est importante. L'activité de ${ }^{106} \mathrm{Ru}-\mathrm{Rh}$ mesurée dans l'algue n'est pas liée à l'activité rejetée par une relation simple. Pendant la période 1975-1990, l'impact des rejets de ${ }^{106} \mathrm{Ru}-\mathrm{Rh}$ a été moins marqué en rade de Cherbourg qu'à Goury. En 1991, par contre, l'impact apparaît plus important en rade de Cherbourg, malgré un effet de dilution accru dans l'eau de cette station (tableau VII, colonne 1). L'évolution des structures chimiques complexes du ruthénium rejeté en mer en 1991 va donc dans le sens d'une plus grande réactivité par rapport à Fucus serratus.

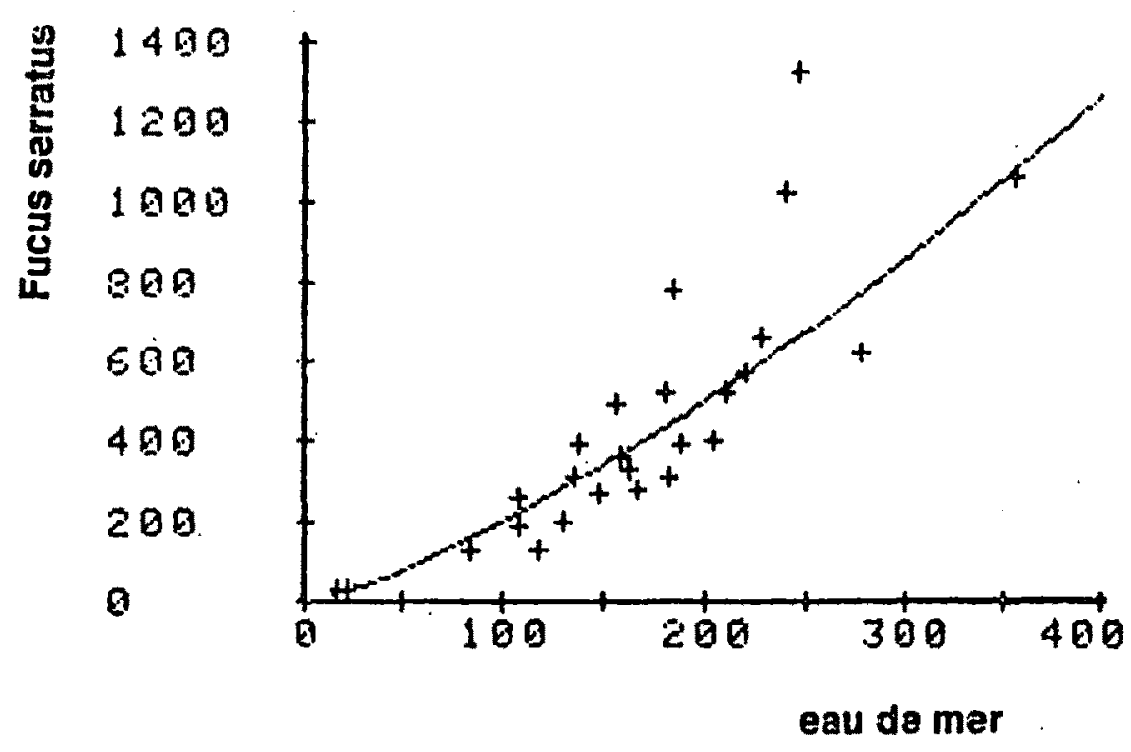

Fig. 6 - Expression des activités moyennes annuelles de 106 Ru-Rh mesurées dans Fucus serratus, en fonction des valeurs obtenues dans l'eau de mer en utilisant le bioxyde de manganèse en milieu basique.

${ }^{106} R u-R h$ annual average activities measured in Fucus serratus vs sea water values obtained using $\mathrm{MnO}_{2}$ in basic medium.

Ce comportement est à rapprocher du celui que nous avons mis en évidence par rapport à $\mathrm{MnO}_{2}$. La figure 6 exprime la relation entre l'activité moyenne annuelle de ${ }^{106} \mathrm{Ru}-\mathrm{Rh}(y)$ mesurée dans l'algue sèche (Tab. VI) et celle déterminée dans l'eau de mer $(x)$, en utilisant $\mathrm{MnO}_{2}$ à $\mathrm{pH}$ basique (Tab. V). Chaque point du diagramme correspond à 36 mesures de radioactivité $(24$ relatives à l'eau et 12 relatives à Fucus serratus). L'expression mathématique est de la 
INCIDENCES DES PROCÉDÉS D'ÉPURATION SUR 106RU DANS L'ENVIRONNEMENT MARIN

TABLEAU IX

Bilan de l'impact $Q$ des rejets de ${ }^{106} \mathbf{R u}-\mathrm{Rh}$ sur l'activité de l'algue Fucus serratus en rade de Cherbourg et à Goury, pendant la période 1975-1991 :

Assessment of the impact $Q$ of ${ }^{106} \mathrm{Ru}-\mathrm{Rh}$ releases on Fucus serratus activity in Cherbourg harbour and at Goury, 1975-1991

\begin{tabular}{|ccc|}
\hline & Rade de Cherbourg & Goury \\
\hline 1975 & 0,75 & 1,36 \\
1976 & 0,49 & 1,08 \\
1977 & 0,61 & 1,35 \\
1978 & 0,36 & 0,96 \\
1979 & 0,69 & 1,14 \\
1980 & 0,33 & 1,81 \\
1981 & 0,73 & 2,85 \\
1982 & 0,90 & 1,22 \\
1983 & 0,77 & 1,97 \\
1984 & 0,52 & 1,46 \\
1985 & 0,36 & 0,65 \\
1986 & 0,48 & 0,81 \\
1987 & 0,26 & 0,38 \\
1988 & 0,39 & 0,75 \\
1989 & 0,24 & 0,57 \\
1990 & 0,44 & 0,52 \\
1991 & 1,25 & 1,15 \\
\hline
\end{tabular}

$Q=\frac{\text { Activité }{ }^{106} \mathrm{Ru}-\mathrm{Rh} \text { mesurée dans Fucus serratus }\left(\mathrm{Bq} \mathrm{kg}^{-1} \text { sec }\right)}{\text { Activité des rejets en }{ }^{106} \mathrm{Ru}-\mathrm{Rh}\left(\mathrm{GBq} \mathrm{an}^{-1}\right)}$

forme $(y)=0,429(x)^{1,332}$ et le coefficient de corrélation correspondant $\left(r^{2}\right)$ est de 0,893. L'activité de Fucus serratus tend vers une valeur nulle lorsque la concentration des formes hydrolysées du ruthénium tend vers zéro. Les formes hydrolysées des complexes de ${ }^{106} \mathrm{Ru}$ sont donc des formes privilégiées dans les phénomènes d'échange avec l'algue Fucus serratus, dans le milieu marin naturel.

Le tableau $X$ présente le bilan des facteurs de concentration de l'algue fraîche par rapport aux formes hydrolysées des complexes de ${ }^{106} \mathrm{Ru}$ déterminées dans l'eau de mer (Tab. V), avec la méthode décrite par Guéguéniat et al. [16]. Les valeurs sont calculées à partir du bilan de radioactivité de l'algue (Tab. VI), en tenant compte d'une teneur moyenne de Fucus serratus en matière sèche de $21 \% \pm 2 \%$ par rapport au produit frais. La moyenne des facteurs de concentration est de $458 \pm 289$ à Cherbourg et de $585 \pm 213$ à Goury, 
pendant les périodes où les mesures ont été réalisées. On peut donc considérer que, par rapport aux formes hydrolysées des complexes de ${ }^{106} \mathrm{Ru}$, le facteur de concentration de Fucus serratus est de $500 \pm 350$.

\section{Conclusions}

Le comportement chimique du ${ }^{106} \mathrm{Ru}$ dans l'environnement marin proche de l'émissaire de l'usine de retraitement des combustibles irradiés de La Hague est révélateur de l'évolution des techniques d'épuration appliquées aux effluents pendant la période 1966-1991.

De 1966 à 1988-1989, l'épuration des effluents liquides a été basée sur des méthodes d'extraction des radionucléides en phase aqueuse. L'optimisation du traitement de ${ }^{106} \mathrm{Ru}$ par le sulfure de cobalt en milieu basique a été obtenue en 1983. A partir de 1990, un procédé "voie sèche" lui a été adjoint, les effluents des ateliers de dissolution des combustibles irradiés et du premier cycle d'extraction U-Pu ont été concentrés par évaporation dans le but de les vitrifier.

TABLEAU $X$

Bilan des facteurs de concentration de Fucus serratus, exprimés par rapport à l'algue fraîche et aux formes hydrolysées des complexes de ${ }^{106} \mathbf{R u}$, à l'entrée ouest de la rade de Cherbourg, entre 1975 et 1991 et à Goury, entre 1982 et 1991.

Fresh Fucus serratus concentration factors $v$ s hydrolysis products of ${ }^{106} \mathbf{R u}$ complexes, Cherbourg harbour west entry, 1975-1991, and Goury, 1982-1991.

\begin{tabular}{|ccc|}
\hline & Rade de Cherbourg & Goury \\
\hline 1975 & $470 \pm 35$ & \\
1976 & $390 \pm 65$ & \\
1977 & $430 \pm 70$ & \\
1978 & $365 \pm 55$ & \\
1979 & $520 \pm 40$ & \\
1980 & $500 \pm 70$ & \\
1981 & $655 \pm 90$ & \\
1982 & $890 \pm 130$ & $630 \pm 55$ \\
1983 & $605 \pm 75$ & $1130 \pm 90$ \\
1984 & $485 \pm 80$ & $892 \pm 70$ \\
1985 & $365 \pm 55$ & $545 \pm 40$ \\
1986 & $435 \pm 70$ & $605 \pm 45$ \\
1987 & $385 \pm 65$ & $415 \pm 45$ \\
1988 & $325 \pm 50$ & $590 \pm 95$ \\
1989 & $340 \pm 75$ & $480 \pm 75$ \\
1990 & $370 \pm 50$ & $230 \pm 40$ \\
1991 & $255 \pm 65$ & $325 \pm 85$ \\
\hline
\end{tabular}


Les rejets normalisés de ${ }^{106} \mathrm{Ru}-\mathrm{Rh}$ permettent de quantifier l'efficacité des procédés d'épuration appliqués aux effluents. En 1975, l'efficacité du traitement correspondait à un rejet annuel de 9,6 TBq par kilogramme de ruthénium. La maîtrise du procédé d'épuration par le sulfure de cobalt a contribué à réduire les valeurs des rejets annuels normalisés à $1,3 \mathrm{TBq}$ en 1983 et $0,6 \mathrm{TBq}$ en 1989. Les mises en service successives de la "voie sèche" et de la station STE3 de traitement des effluents liquides ont permis d'abaisser, en 1992, les rejets normalisés de $106 \mathrm{Ru}-\mathrm{Rh}$ à $0,016 \mathrm{TBq} / \mathrm{kg}$ de ruthénium. Par rapport à l'année 1975 prise en référence, l'efficacité des effluents radioactifs a donc été multipliée par 7,4 en 1983, par 16 en 1989, par 600 en 1992.

Les techniques d'épuration appliquées aux effluents ont influencé la nature physico-chimique de ${ }^{106} \mathrm{Ru}$ dans les rejets. Entre 1983 et 1989, l'amélioration du procédé au sulfure de cobalt a eu pour conséquence d'augmenter la part des complexes forts du ruthénium. A partir de 1990, le procédé d'épuration par "voie sèche" appliqué à ces complexes forts a contribué à réduire leur participation dans les rejets. Le ruthénium s'y trouve alors à l'état de complexes plus facilement hydrolysés.

Depuis la mise en service de l'usine de La Hague, le comportement général du ruthénium dans l'eau de mer du milieu naturel a montré le rôle déterminant des caractères physico-chimiques de l'élément au moment où il est introduit dans le milieu marin. Les complexes forts représentatifs de la période 19831989 manifestent une tendance peu marquée à l'hydrolyse. En 1991, près de la moitié du ruthénium dispersée en mer a été hydrolysé pendant le transit des masses d'eau entre les stations de Goury et Cherbourg ( 1 à 3 semaines pour une distance de $30 \mathrm{~km}$ ).

Une activité maximale de $1910 \mathrm{~Bq} \mathrm{~kg}^{-1} \mathrm{sec}$ dans Fucus serratus a été déterminée, en 1981, à Goury. Dans la rade de Cherbourg située à $35 \mathrm{~km}$ du point de rejet, l'activité la plus élevée ( $780 \mathrm{~Bq} \mathrm{~kg}^{-1} \mathrm{sec}$ ) a été enregistrée en 1982 . Au cours des années suivantes, la concentration en ${ }^{106} \mathrm{Ru}-\mathrm{Rh}$ dans l'algue a diminué. En 1991, à Goury, elle a été divisée par 72 par rapport à 1981 et dans la rade de Cherbourg, elle a été divisée par 27 par rapport à 1982.

Les produits d'hydrolyse de ${ }^{106} \mathrm{Ru}$ sont des formes chimiques privilégiées d'échange avec l'algue Fucus serratus. La technique d'analyse de ${ }^{106} \mathrm{Ru}-\mathrm{Rh}$ par $\mathrm{MnO}_{2}$ à $\mathrm{pH}$ basique [16] permet la détermination de ces produits d'hydrolyse du ruthénium. Elle constitue une méthode fiable pour évaluer la biodisponibilité du ${ }^{106} \mathrm{Ru}$ de l'eau de mer vis-à-vis de Fucus serratus.

Le facteur de concentration de l'algue fraîche déterminé dans le milieu naturel est de l'ordre de $500 \pm 350$, par rapport à ces formes hydrolysées du ruthénium. 


\section{Remerciements}

Les auteurs expriment leur profonde gratitude à l'égard de M. Henri Métivier pour ses précieux conseils et son aide dans la rédaction finale de l'article.

Les personnels du Groupe d'études atomiques de la marine nationale de Cherbourg ont apporté à ce travail une contribution et une compétence sans faille. Qu'ils en soient également remerciés.

\section{RÉFÉRENCES}

[1] AGENCE INTERNATIONALE DE L'ÉNERGIE ATOMIQUE (AIEA). Sediment kds and concentration factors for radionuclides in the marine environment (Technical reports series $n^{\circ} 247$ ). Vienna : AIEA, 1985, 24-44.

[2] ANCELlin J., GUEGUENIAT P., GERMAIN P. - In : Radioécologie marine. Etude du devenir des radionucléides rejetés en milieu marin. Paris : Eyrolles, 1979, 118-119.

[3] AVtokratova, T. D. - Analytical chemistry of ruthenium. Ann Arbor : Ann Arbor-Humphrey science publishers, 1969.

[4] BERTON, Y., CHAUVET, P. - Procédé de décontamination d'effluents radioactifs, notamment en antimoine et en ruthénium. (Institut national de la protection industrielle, Brevet d'invention $\mathrm{n}^{\circ} 2346$ 817). Paris : Imprimerie nationale, 1976.

[5] BLASIUS E., LUXENBURGER H.J., NEUMANN W. - Ruthenium nitrosyl complexes in radioactive waste solutions of reprocessing plants. Radiochim. Acta, 1984, 36, 149-153.

[6] BOUST D., HAIRIE A., FRAIZIER A., BARON Y. - Nouvelle méthode statistique prédictive appliquée à l'étude de la dispersion des radioéléments dans l'environnement marin. Oceanol. Acta, 1994 (à paraître).

[7] FLETCHER J.M., JENKINS I.L., LEVER F.M., MARTIN F.S., POWELL A.R., TODD R. - Nitrato and nitro complexes of nitrosylruthenium. J. Inorg. Nucl. Chem., 1955, 1, 378-401.

[8] GANDON R. - Les mécanismes d'adsorption des espèces minérales cationiques et anioniques sur les hydroxydes polymères du fer ferrique et les produits d'oxydation du fer ferrique et les produits d'oxydation du fer ferreux en milieu aqueux. Rapport CEA-R-5238, 1983.

[9] GANDON R., GUEGUENIAT P. - Preconcentration of ${ }^{125} \mathrm{Sb}$ onto $\mathrm{MnO}_{2}$ from seawater samples for gamma-ray spectrometric analysis. Radiochim. Acta, 1992, 57, 159-164.

[10] GANDON R., BOUST D., BEDUE O. - Ruthenium complexes originating from the Purex process : coprecipitation with copper ferrocyanides via ruthenocyanide formation. Radiochim Acta, 1993, 61, 41-45.

[11] GERMAIN P. MASSON M., BARON Y. - Etude de la répartition des radionucléides émetteurs gamma chez des indicateurs biologiques littoraux des côtes de la Manche et de la mer du Nord de février 1976 à février 1978. Rapport CEA-R-5017, 1979.

[12] GERMAIN P., MASSON M., BARON Y. - Contribution aux recherches sur les mouvements des éléments à l'état de traces dans les eaux côtières de la Manche, par l'étude de la distribution spatiale de traceurs radioactifs industriels dans les moules et les fucus. Rapport CEA-R-5534, 1990. 
[13] GERMAIN P., SALOMON J. C. - Transport d'éléments à l'état de traces dans les eaux côtières de la Manche : étude de la distribution spatiale d'un traceur radioactif $\left({ }^{106} \mathrm{Ru}-\mathrm{Rh}\right)$ dans les moules et les fucus. In : Radionuclides in the study of marine processes, Norwich UK, 10-13 September 1991 (Kershaw J.P., Woodhead D.S., Eds.). New-York : Elsevier, 1991, 364.

[14] GOLDBERG E.D., KOIDE M. - Understanding the marine chemistries of the platinum group metals. Marine Chem., 1990, 30, 249-257.

[15] GUEGUENIAT P. - Comportement physico-chimique du ruthénium de fission dans le milieu marin. Rapport CEA-R-4644, 1974.

[16] GUEGUENIAT P., GANDON R., LUCAS Y. - Determination of radionuclides of $\mathrm{Ce}, \mathrm{Co}, \mathrm{Fe}, \mathrm{Ru}, \mathrm{Zn}$ and $\mathrm{Zr}$ in seawater by preconcentration of colloidal manganese dioxide. Application to the determination of low-level ruthenium 106. In : Reference methods for marine radioactivity studies II (Technical reports series $\mathrm{n}^{\circ} 169$ ). Vienne : AIEA, 1975, 137-145.

[17] GUEGUENIAT P., BAILLY du BOIS P., GANDON R., SALOMON J.C., LEON R. - Spatial and temporal distribution of ${ }^{125} \mathrm{Sb}$ used to trace pathways and transit times of waters entering the North sea from the English Channel. Estuar. Coast Shelf Sci., 1993, 36, 477-494.

[18] HOLDER J.V. - A review of the solvent extraction process chemistry of fission products. Radiochim. Acta, 1978, 25, 171-180.

[19] MALVYN McKIBBEN J. Chemistry of the Purex process. Radiochim. Acta, 1984, 36, 3-15.

[20] MORGAN J., STUMM W. - Colloid-chemical properties of manganese dioxide. J. Colloid Sci., 1964, 19, 347-359, d'après GADDE R.R., LAITINEN H.A. - Studies of heavy metal adsorption by iron and manganese oxides. Anal. Chem. 1974, 46, 20222026.

[21] MURRAY J.W. - The surface chemistry of hydrous manganese dioxide. J. Colloid Interface Sci., 1974, 46, 357-371.

[22] SALOMON J.C., GUEGUENIAT P., ORBI A., BARON Y. - A Lagrangian model for long term tidally induced transport and mixing. Verification by artificial radionuclide concentrations. In : Radionuclides, a tool for oceanography (Guary J.C., Guéguéniat P., Pentreath R.J., Eds.). New York : Elsevier, 1988, 384-394.

[23] SALOMON J.C., BRETON M. - Courants résiduels de marée dans la Manche. Oceanol. Acta, 1991, 11, 47-53.

[24] SALOMON J.C., GUEGUENIAT P., BRETON M. - Mathematical model of $125 \mathrm{Sb}$ transport and dispersion in the Channel. In : Radionuclides in the study of marine processes (Kershaw P.J., Woodhead D.S., Eds.). New York : Elsevier, 1991, 74-83.

[25] SATO T. - Chemical species of fission products ruthenium in the solution of irradiated uranium dioxide. Radiochim. Acta, 1989, 47, 219-228.

[26] SERVICE CENTRAL DE PROTECTION CONTRE LES RADIATIONS IONISANTES (SCPRI). Résultats des contrôles spéciaux consécutifs à l'accident de Tchernobyl et cartes de la radioactivité par régions, mai-juin 1986 (Rapport RM-6$1986 ;$ Pt.2) Le Vésinet : SCPRI, 1986. 